\title{
Urine nerve growth factor (NGF) level, bladder nerve staining and symptom/problem scores in patients with interstitial cystitis
}

\author{
Senol Tonyali ${ }^{1}$, A-F , Deniz Ates ${ }^{2, A-F}$, Filiz Akbiyik' ${ }^{3, A-F}$, Duygu Kankaya ${ }^{4, A-F}$, Dilek Baydar ${ }^{2, A-F}$, Ali Ergen ${ }^{1, A-F}$ \\ ${ }^{1}$ Department of Urology, Hacettepe University School of Medicine, Ankara, Turkey \\ ${ }^{2}$ Department of Pathology, Hacettepe University School of Medicine, Ankara, Turkey \\ ${ }^{3}$ Department of Medical Biochemistry, Hacettepe University School of Medicine, Ankara, Turkey \\ ${ }^{4}$ Department of Pathology, Ankara University School of Medicine, Ankara, Turkey \\ A - research concept and design; $\mathrm{B}$ - collection and/or assembly of data; $\mathrm{C}$ - data analysis and interpretation; \\ $D$ - writing the article; $E$ - critical revision of the article; $F$ - final approval of the article
}

Address for correspondence

Senol Tonyali

E-mail:dr.senoltonyali@gmail.com

\section{Funding sources}

This study was funded by the Hacettepe University

Scientific Research Projects Coordination Unit (Ankara, Turkey).

\section{Conflict of interest}

None declared

\section{Acknowledgements}

We would like to thank Anil Barak, MD for the statistical analysis.

Received on September 2, 2016 Reviewed on December 30, 2016 Accepted on February 28, 2017

DOI

10.17219/acem/69231

\section{Copyright}

Copyright by Author(s)

This is an article distributed under the terms of the

Creative Commons Attribution Non-Commercial License

(http://creativecommons.org/licenses/by-nc-nd/4.0/)

\begin{abstract}
Background. Interstitial cystitis (IC)/bladder pain syndrome (BPS) is a challenging disease, affecting thousands of people all around the world, especially women. Although there have been numerous theories regarding IC/BPS etiology, the physiopathology of the disease still remains unclear and there is a lack of certain treatment.

Objectives. The aim of the study was to assess the role of nerve fibers and nerve growth factor (NGF) in the etiopathogenesis of IC/BPS symptoms by demonstrating if there is a correlation between urine NGF levels, amount of peripheral nerves in bladder mucosa and symptom severity.

Material and methods. A prospective clinical study was conducted with 15 IC/BPS patients and 18 controls. Urine NGF levels were measured by enzyme-linked immunosorbent assay (ELISA). Bladder punch biopsies were obtained from 15 IC/BPS patients and 9 controls. Immunohistochemistry was performed for S-100 to highlight peripheral nerve twigs in bladder mucosa. The O'Leary-Sant Interstitial Cystitis Symptom and Problem Index (OSICSPI) was used to assess symptom severity and effects of the disease on the patients' life.

Results. NGF normalized to urine creatinine (NGF/Cr) levels in IC/BPS patients were significantly higher than in controls, $0.34 \pm 0.22$ and $0.09 \pm 0.08 \mathrm{pg} / \mathrm{mL}$ : $\mathrm{mg} / \mathrm{dL}$, respectively $(p<0.001)$. The mean symptom score in IC patients was $12.27 \pm 2.4$ (median: 12) and the mean problem score was $10.9 \pm 2.3$ (median: 12). The mean mucosal nerve (S-100 stained) area in the IC/BPS group was significantly higher than in the controls, $2.53 \pm 1.90$ vs $1.0 \pm 0.70$, respectively $(p=0.018$ ). In correlation analyses, the NGF/Cr level in IC/BPS patients was found significantly correlated with the O'Leary-Sant IC Symptom and Problem Index scores independently ( $p=0.001$ and $p=0.028$, respectively).

Conclusions. NGF seems to be a promising biomarker in IC/BPS. It may help clinicians in diagnoses and patient follow-up. Thus, unnecessary, expensive and invasive tests, interventions and treatments might be avoided.
\end{abstract}

Key words: nerve growth factor, bladder pain syndrome/interstitial cystitis, nerve staining 


\section{Introduction}

Interstitial cystitis (IC)/bladder pain syndrome (BPS) is a challenging disease that affects thousands of people worldwide, especially women. IC/BPS is characterized by pelvic pain, pressure and discomfort perceived to be related to the urinary bladder, associated with lower urinary tract symptoms (LUTS) in the absence of urinary infection and other obvious pathology. ${ }^{1}$

Although there are numerous theories in regard to IC/BPS etiology, its physiopathology still remains unclear and there is a lack of certain treatment. ${ }^{2}$

Various diagnostic tests and attempts at treatment lead to a substantial economic burden as well as patient distress. Thus, using biomarkers in diagnosis has garnered greater interest in the last decade. ${ }^{3}$ Nerve growth factor (NGF) is one of these.

In the present study, we aimed to assess the role of nerve fibers and nerve growth factor (NGF) in the etiopathogenesis of IC/BPS symptoms by demonstrating if there is a correlation between urine NGF levels, amount of peripheral nerves in bladder mucosa and symptom severity. This might open a new horizon for disease diagnosis and yield novel treatment alternatives.

\section{Material and methods}

After local ethics committee approval, a prospective clinical study was conducted between March, 2014 and February, 2015 with 15 patients diagnosed with IC/BPS according to AUA 2011 criteria $^{1}$ and 18 controls. Written informed consent was obtained from patients and controls. Previous intake of oral medications or intravesical instillations for the treatment of IC/BPS was not exclusionary. The sole inclusion criterion for the patient group was to be symptomatic. The control group consisted of patients who underwent cystoscopy for previous bladder tumor surveillance or LUTS. Urinary tract infections were excluded by means of urine culture in all cases. The O'Leary-Sant Interstitial Cystitis Symptom and Problem Index (OSICSPI) was used to assess the symptom severity and effects of the disease on the patient's life. ${ }^{4}$

Urine samples and bladder biopsies were obtained from all patients with IC/BPS. Although urine samples were obtained from all controls, bladder biopsies could be obtained from 9 controls. Among these 9 controls, 5 were on follow-up for bladder tumor (with no evidence of disease for at least 1 year) and 4 were the patients who underwent cystoscopy for LUTS.

Midstream clean catch urine specimens were collected from all patients and controls. Urine samples were put on ice and promptly transferred to a biochemistry laboratory for NGF and creatinine $(\mathrm{Cr})$ measurement. The samples were centrifuged at $1500 \mathrm{rpm}$ for $20 \mathrm{~min}$ at $4^{\circ} \mathrm{C}$. The supernatant was taken into Eppendorf tubes and stored at $-80^{\circ} \mathrm{C}$ until NGF measurement after $3 \mathrm{~mL}$ was separated for urinary $\mathrm{Cr}$ measurement.

The urine NGF level was measured by the enzyme-linked immunosorbent assay (ELISA) method using a NGF specific ELISA kit (Cloud-Clone Corp., Houston, USA). Assays were performed in accordance with the manufacturer's instructions. All samples were run in duplicate, and then the results were averaged. Afterwards, urine NGF levels were normalized via urine creatinine concentration (NGF/Cr).

Bladder punch biopsies were obtained by means of cystoscopy under local or general anesthesia from the lesion sites or suspicious areas of the bladder, if present, otherwise from the lateral wall in IC/BPS and from the normal mucosa in controls. Biopsy specimens were put in $4 \%$ formaldehyde solution for fixation and submitted to a pathology laboratory. After routine overnight tissue processing, paraffin blocks were prepared and $5 \mu \mathrm{m}$ sections were obtained for hematoxylin and eosin (H\&E) staining and microscopic evaluation. Immunohistochemistry was performed for S-100 to highlight peripheral nerve twigs in bladder mucosa. Immunohistochemical staining was carried out according to standard procedures using a polymer detection system (Cat. No. DS9800, Leica Biosystems, Wetzlar, Germany) and a BOND-MAX automated immunostainer. S-100 primary antibody (Dako, Glostrup, Denmark) was applied at a dilution of $1 / 400$.

\section{Evaluation of nerve density using S-100 immunohistochemistry}

\section{Visual analysis}

The amount of peripheral nerve twigs in bladder mucosa was evaluated and scored semiquantitatively by a pathologist (DEB) under the microscope on a range of $0-3$ as follows: $0=<1$ mean nerve twig $(\mathrm{NT})$ per high power field (hpf) ${ }^{-1} ; 1=1-2 \mathrm{NTs} / \mathrm{hpf}^{-1} ; 2=3-4 \mathrm{NTs} / \mathrm{hpf}^{-1}$, and $3=\geq 5 \mathrm{NTs} / \mathrm{hpf}^{-1}$ at various thickness (Fig. 1). Subjects were divided into 3 groups - no staining, mild staining and obvious staining - according to the aforementioned scores (no staining $=$ score 0 ; mild staining $=$ score 1 ; obvious staining $=$ score 2 or 3 ).

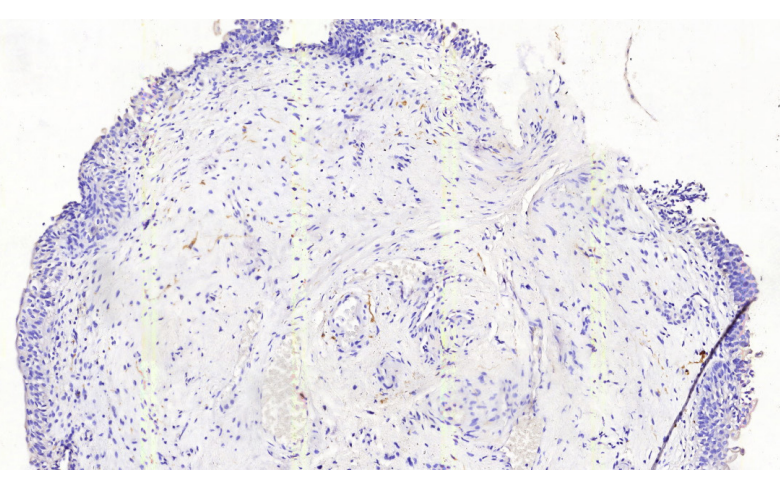

Fig. 1. Microscopic examination of urinary bladder specimen with no nerve staining at magnification $\times 10$ with $H \& E$ and S-100 stains 


\section{Image analyzer}

The strength of the immunohistochemical staining of urinary bladder was assessed using a computer-assisted image analyzer (Pannoramic250 Flash II; 3DHistech, Budapest, Hungary) and the density of nerve fibers was determined automatically.

\section{Statistical analysis}

Mean \pm SD and median values were used to express quantitative measurements. Numbers and percentages were given for qualitative measurements. The ShapiroWilk test was used to determine whether the measurements distributed normally or not. The Mann-Whitney $\mathrm{U}$ test was used to compare numerical values between independent groups. For the comparison of the qualitative characteristics of the 2 groups, cross table analysis, $X^{2}$ Fisher's exact test and the Fisher-Freeman-Halton test were applied. Statistical analysis was performed via IBM SPSS statistics v. 21 (IBM Corp., Armonk, USA) and p $<0.05$ was considered to indicate statistical significance.

\section{Results}

A total of 33 patients were included in the study, of whom 15 were patients with IC/BPS and 18 were controls. All patients with IC/BPS were females, whereas among the 18 controls, 12 were males and 6 were females. The mean age of the IC/BPS patients was $52 \pm 9.1$ years, while that of the control group was $46.9 \pm 19.2$ years. There was no statistically significant difference between IC/BPS patients and controls in terms of age $(p>0.5)$. Patient demographics are given in Table 1.
The mean urine NGF level normalized to the urine Cr level (NGF/Cr) in IC/BPS patients was significantly higher than in controls, $0.34 \pm 0.22 \mathrm{pg} / \mathrm{mL}: \mathrm{mg} / \mathrm{dL}$ and $0.09 \pm 0.08 \mathrm{pg} / \mathrm{mL}: \mathrm{mg} / \mathrm{dL}$, respectively ( $<<0.001)$. The mean symptom score in IC/BPS patients was $12.27 \pm 2.4$ (median: 12 ) and the mean problem score was $10.9 \pm 2.3$ (median: 12) (Table 1).

The biopsies of the controls displayed only a few nerve twigs in bladder mucosa in general (Fig. 1). On the other hand, the majority of IC/BPS patients (93.3\%) had an increased number of mucosal nerve twigs (Fig. 2), visually scored as 2 or 3 in S-100 stained slides (Table 2). This difference between the control and study groups was statistically significant $(\mathrm{p}<0.05)$.

Image analysis showed similar results concerning the difference in the amounts of nerve twigs between the 2 groups (Table 1). The mean mucosal nerve (S-100 stained) area in the IC/BPS group was significantly higher than in the controls, $2.53 \pm 1.90$ vs $1.0 \pm 0.70$, respectively ( $\mathrm{p}=0.018$ ).

In correlation analyses (Table 3), the normalized NGF level (NGF/Cr) in IC/BPS patients was found significantly and

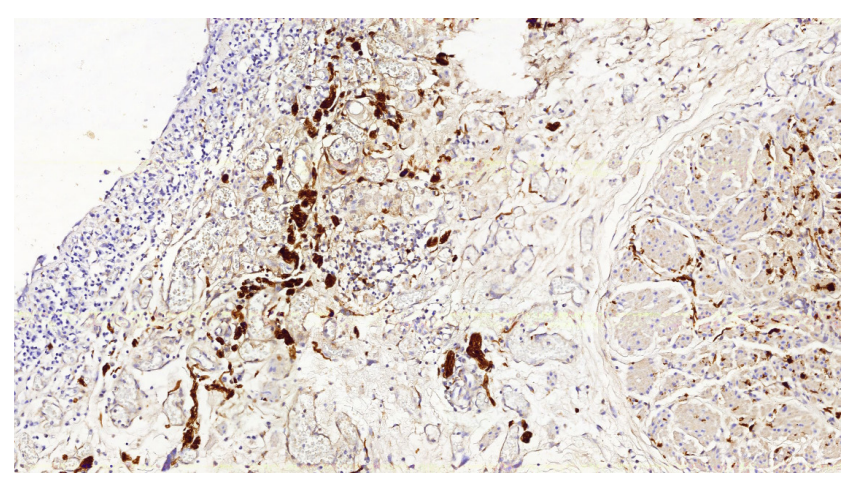

Fig. 2. Microscopic examination of urinary bladder specimen with obvious nerve staining at magnification $\times 10$ with $\mathrm{H} \& \mathrm{E}$ and S-100 stains

Table 1. Patient age, NGF, automated nerve density and questionnaire scores

\begin{tabular}{|l|c|c|}
\multicolumn{1}{c}{ Variable } & \multicolumn{2}{c|}{ Group } \\
\cline { 2 - 3 } & IC/BPS $(\mathrm{n}=15)$ & Control $(\mathrm{n}=18)$ \\
\hline Age (mean \pm SD) [years] & $52 \pm 9.1$ & $46.9 \pm 19.2$ \\
\hline NGF/Cr (mean \pm SD) $[\mathrm{pg} / \mathrm{mL}: \mathrm{mg} / \mathrm{dL}]$ & $0.34 \pm 0.2$ & $0.09 \pm 0.08$ \\
\hline Automated nerve density (mean S100 (+) area \%) & $2.53 \pm 1.9$ & $1.0 \pm 0.7^{*}$ \\
\hline O'Leary-Sant IC Symptom Index Score & $12.2 \pm 2.4$ & - \\
\hline O'Leary-Sant IC Problem Index Score & $10.9 \pm 2.3$ & - \\
\hline
\end{tabular}

* $n+9$.

Table 2. Distribution of patients according to nerve staining

\begin{tabular}{|l|c|c|}
\multicolumn{1}{|c|}{ Visual nerve staining density groups } & \multicolumn{2}{c|}{ Group } \\
\cline { 2 - 3 } & IC/BPS & control \\
\hline Group 1. Number of patients with no staining & 1 & 6 \\
\hline Group 2. Number of patients with mild staining & 10 & 3 \\
\hline Group 3. Number of patients with obvious staining & 4 & 0 \\
\hline Total & 15 & 9 \\
\hline
\end{tabular}


Table 3. Correlation analyses

\begin{tabular}{|c|c|c|c|c|}
\hline Variable & Spearman's rho & $\begin{array}{l}\text { O'Leary-Sant IC Symptom } \\
\text { Index Score }\end{array}$ & $\begin{array}{l}\text { O'Leary-Sant IC Problem } \\
\text { Index Score }\end{array}$ & NGF/Cr level \\
\hline \multirow{2}{*}{ NGF/Cr level } & correlation coefficient & 0.777 & 0.567 & - \\
\hline & significance (2-tailed) & 0.001 & 0.028 & - \\
\hline \multirow{2}{*}{ Visual nerve density } & correlation coefficient & 0.577 & 0.640 & 0.325 \\
\hline & significance (2-tailed) & 0.024 & 0.010 & 0.237 \\
\hline \multirow{2}{*}{ Automated nerve density } & correlation coefficient & 0.420 & 0.650 & 0.265 \\
\hline & significance (2-tailed) & 0.119 & 0.009 & 0.341 \\
\hline
\end{tabular}

independently correlated with the O'Leary-Sant IC Symptom and Problem Index scores $(p=0.001$ and $p=0.028$, respectively). Visually analyzed mucosal nerve density and image analyzed nerve density were found to be correlated with the O'Leary-Sant IC Problem Index ( $\mathrm{p}=0.01$ and $\mathrm{p}=0.009$, respectively). However, image analyzed nerve density was not correlated with the O'Leary-Sant IC Symptom Index ( $\mathrm{p}=0.119)$, whereas visually analyzed nerve density was $(p=0.024)$. Furthermore, no correlation was observed between nerve density and NGF/Cr levels ( $p=0.34)$.

\section{Discussion}

IC/BPS is a debilitating, chronic disease characterized by pelvic pain, discomfort and pressure perceived to be related to the urinary bladder and accompanied by at least one LUTS-like urgency or frequency. ${ }^{5}$ The prevalence of IC/BPS is higher in females compared to males, $52-500 / 100,000$ vs $8-41 / 100,000$ and its incidence is estimated to be $1.2 / 100,000 .^{6}$

IC/BPS etiology is still unknown which leads to inaccurate diagnostic tests and treatment modalities. It has been thought to be associated with several conditions such as chronic fatigue syndrome, fibromyalgia, irritable bowel syndrome, temporomandibular junction disorder, chronic pelvic pain syndrome, vulvodynia, migraine, sicca syndrome, allergies, asthma, and depression. ${ }^{7}$ Many theories have been suggested in respect of IC/BPS pathogenesis, which consists of increased urinary bladder permeability, abnormal neuronal function, mast cell activation, autoimmunity, glycosaminoglycan (GAG) layer defect, infection, and urinary toxic and anti-proliferative agents. ${ }^{6,8-11}$

The first study which focused on the nerve fibers in IC/BPS was conducted by Hand in 1949. In that study, he found submucosal nerve fiber increase in the urinary bladders of IC/BPS patients and concluded that nerve fibers within closed mast cells might have a role in disease pathogenesis. ${ }^{12}$ In concordance with Hand, Christmas et al. also observed nerve fiber proliferation in the suburothelium and detrusor muscle layer of the urinary bladder of IC/BPS patients compared to controls and other patients with chronic cystitis. ${ }^{13}$ Pang et al. showed an increase in nerve fibers containing substance-P in the submucosa of IC/BPS patients but not in the detrusor. They suggested that substance-P, a neuropeptide secreted from sensorial nerve endings, might act in pain pathophysiology as well as triggering mast cell secretion. ${ }^{14}$

Hofmeister et al. also attempted to establish a diagnostic algorithm for IC/BPS by using quantitative image analysis and morphometry. They suggested that the neuroimmune process or mediation might be a part of the disease pathogenesis and a count of either mast cells or nerve fibers could be used in IC/BPS diagnosis. ${ }^{15}$

In concordance with the aforementioned studies, we found the mucosa of the urinary bladder of IC/BPS patients to have significantly increased nerve fiber proliferation, either visually or image analyzed. None of the patients in the control group demonstrated obvious nerve staining in the urinary bladder in contrast to IC/BPS patients, which may support the role of a neural process in IC/BPS etiopathogenesis.

Studies conducted in recent years have shown increased levels of NGF in the urothelium, where the sensorial nerve fibers end, in patients with IC/BPS, idiopathic urgency and chronic cystitis. NGF was thought to be responsible for hyperalgesia in the absence of inflammation. NGF was assumed to act via a direct effect on sensorial nerve fiber endings or increasing sensorial neuropeptides, substance-P or calcitonin-gene-related peptide. ${ }^{16}$ In another study, NGF mRNA was found significantly increased in bladder tissues of IC/BPS patients compared to controls, and decreased to normal levels as controls after botulinum toxin A injection. ${ }^{17}$ A similar study based on urinary NGF again showed that a decrease in the NGF level could be correlated with treatment response in some IC/BPS patients. ${ }^{18}$ Many studies have presented a significant increase of NGF levels in IC/BPS and neurogenic overactive bladder, but stressed that further studies were mandatory to determine its role in the pathogenesis. ${ }^{19}$

Some researchers investigated both serum and urine NGF in IC/BPS patients, but no correlation was found between urine and serum NGF levels. Moreover, no association was observed between an elevated serum NGF level and disease severity. It was suggested that an elevated serum NGF level might be a consequence of comorbidities 
of IC/BPS patients rather than the cause of IC/BPS. Elevated urinary NGF levels were deemed to support the role of chronic inflammation in the disease pathogenesis. ${ }^{20}$

In a recent study, inflammation intensity in the urinary bladder was shown to be significantly correlated with suburothelial NGF levels and transient receptor potential cation channel subfamily V member 1 (TRPV1)-immunoreactive nerve fibers. Nerve fiber density was also significantly correlated with pain and urgency scores. ${ }^{21}$

In a meta-analysis, NGF and NGF/Cr levels in the urine of patients with IC/PBS were found higher than in controls and lower than in overactive bladder syndrome patients. NGF is thought to be a potentially useful biomarker in IC/ BPS diagnosis and differential diagnosis. NGF was also proposed to be a predictor of specific treatment modalities. ${ }^{3}$ Antibodies against NGF have emerged as a promising treatment choice for IC/BPS patients. ${ }^{22,23}$

In our study, urinary NGF levels in the urine of IC/BPS patients were significantly higher than those of the controls. Furthermore, the normalized urine NGF level (NGF/ $\mathrm{Cr}$ ) in IC/BPS patients was found significantly correlated with the O'Leary-Sant IC Symptom and Problem Index scores independently. This raises the possibility of using NGF to establish disease alleviation or progression on patient follow-up without the need of an invasive test.

Unfortunately, no statistically significant association was observed between nerve staining and NGF levels, which we believe to be the consequence of the small patient population. However, NGF biology and production might also be considered. It has been shown that, aside from nerve cells, many different cell types, such as vascular and smooth muscle cells, produce NGF in the human body. ${ }^{24}$

Our study has some limitations. As previously mentioned, our patient population was small and, whereas all the IC/ BPS patients were female, some of the controls were males. But the results were similar if we excluded the male controls. Some of the control patients had a history of bladder tumor, so we preferred patients with no evidence of tumor for at least 1 year to prevent the possible effect of the bladder tumor on bladder nerve staining and urine NGF levels.

\section{Conclusions}

NGF appears to be a promising biomarker in patients with IC/BPS. The correlation of NGF and disease symptom/problem scores might help clinicians in diagnosis and patient follow-up, so that unnecessary, expensive and invasive tests, interventions and treatments might be avoided. Additional randomized controlled studies with larger patient populations are mandatory to confirm these results.

\section{References}

1. Hanno PM, Burks DA, Clemens JQ, et al. AUA guideline for the diagnosis and treatment of interstitial cystitis/bladder pain syndrome. J Urol. 2011;185;2162-2170.
2. Chennamsetty A, Ehlert MJ, Peters KM, Killinger KA. Advances in diagnosis and treatment of interstitial cystitis/painful bladder syndrome. Curr Infect Dis Rep. 2015;17:454.

3. Qu HC, Zhang W, Yan S, Liu YL, Wang P. Urinary nerve growth factor could be a biomarker for interstitial cystitis/painful bladder syndrome: A meta-analysis. PLoS One. 2014;9:e106321.

4. O'Leary MP, Sant GR, Fowler FJ Jr, Whitmore KE, Spolarich-Kroll J. The interstitial cystitis symptom index and problem index. Urology. 1997;49:58-63.

5. Hanno P, Lin A, Nordling J, et al. Bladder Pain Syndrome Committee of the International Consultation on Incontinence. Neurourol Urodyn. 2010;29:191-198.

6. Davis NF, Brady CM, Creagh T. Interstitial cystitis/painful bladder syndrome: Epidemiology, pathophysiology and evidence-based treatment options. Eur J Obstet Gynecol Reprod Biol. 2014;175:30-37.

7. Warren JW. Bladder pain syndrome/interstitial cystitis as a functional somatic syndrome. J Psychosom Res. 2014;77:510-515.

8. Keay SK, Zhang CO, Shoenfelt J, et al. Sensitivity and specificity of antiproliferative factor, heparin-binding epidermal growth factorlike growth factor, and epidermal growth factor as urine markers for interstitial cystitis. Urology. 2001;57:9-14.

9. Malykhina AP. Neural mechanisms of pelvic organ cross-sensitization. Neuroscience. 2007;149:660-672.

10. Sant GR, Kempuraj D, Marchand JE, Theoharides TC. The mast cell in interstitial cystitis: Role in pathophysiology and pathogenesis. Urology. 2007;69:34-40.

11. Warren JW. Is interstitial cystitis an infectious disease? Med Hypotheses. 1994;43:183-186.

12. Hand JR. Interstitial cystitis: Report of 223 cases (204 women and 19 men). J Urol. 1949;61:291-310.

13. Christmas TJ, Rode J, Chapple CR, Milroy EJ, Turner-Warwick RT. Nerve fibre proliferation in interstitial cystitis. Virchows Arch A Pathol Anat Histopathol. 1990;416:447-451.

14. Pang X, Marchand J, Sant GR, Kream RM, Theoharides TC. Increased number of substance $P$ positive nerve fibres in interstitial cystitis. Br J Urol. 1995;75:744-750.

15. Hofmeister MA, He F, Ratliff TL, Mahoney T, Becich MJ. Mast cells and nerve fibers in interstitial cystitis (IC): An algorithm for histologic diagnosis via quantitative image analysis and morphometry (QIAM). Urology. 1997;49:41-47.

16. Lowe EM, Anand P, Terenghi G, Williams-Chestnut RE, Sinicropi DV, Osborne JL. Increased nerve growth factor levels in the urinary bladder of women with idiopathic sensory urgency and interstitial cystitis. Br J Urol. 1997;79:572-577.

17. Liu HT, Kuo HC. Intravesical botulinum toxin A injections plus hydrodistension can reduce nerve growth factor production and control bladder pain in interstitial cystitis. Urology. 2007;70:463-468.

18. Liu HT, Tyagi $P$, Chancellor MB, Kuo HC. Urinary nerve growth factor level is increased in patients with interstitial cystitis/bladder pain syndrome and decreased in responders to treatment. BJU Int. 2009; 104:1476-1481.

19. Jacobs BL, Smaldone MC, Tyagi V, et al. Increased nerve growth factor in neurogenic overactive bladder and interstitial cystitis patients. Can J Urol. 2010;17:4989-4994.

20. Liu HT, Kuo HC. Increased urine and serum nerve growth factor levels in interstitial cystitis suggest chronic inflammation is involved in the pathogenesis of disease. PLoS One. 2012;7:e44687.

21. Liu BL, Yang F, Zhan HL, et al. Increased severity of inflammation correlates with elevated expression of TRPV1 nerve fibers and nerve growth factor on interstitial cystitis/bladder pain syndrome. Urol Int. 2014;92:202-208.

22. Nickel JC, Mills IW, Crook TJ, et al. Tanezumab reduces pain in women with interstitial cystitis/bladder pain syndrome and patients with nonurological associated somatic syndromes. J Urol. 2016;195:942948.

23. Evans RJ, Moldwin RM, Cossons N, Darekar A, Mills IW, Scholfield D. Proof of concept trial of tanezumab for the treatment of symptoms associated with interstitial cystitis. J Urol. 2011;185:1716-1721.

24. Sofroniew MV, Howe CL, Mobley WC. Nerve growth factor signaling, neuroprotection, and neural repair. Annu Rev Neurosci. 2001;24:12171281. 